\title{
Prise en compte des impacts dans la réglementation
}

\author{
par Daniel Renard
}

Chef de la Division Planification et Milieux Aquatiques, Direction Régionale de l'Environnement d'lle-de-France

\section{LES EAUX PLUVIALES : DROIT PUBLIC OU DROIT PRIVÉ?}

Le dispositif législatif existant dans le domaine de l'eau, avant la parution de la loi du 3 janvier 1992, est constitué par une accumulation de textes s'échelonnant de 1898 à 1973, d'application limitée, tant dans leur objet (écoulement des eaux, qualité des eaux, ...) que dans l'espace (cours d'eau domaniaux, non domaniaux, eaux souterraines, ...). De surcroit, ces textes laissent subsister des règles très anciennes, tels que les droits fondés en titre et les usages locaux. De ce fait, plusieurs régimes sont applicables à l'eau, marquant une prédominance plus ou moins grande, tantôt du droit privé, tantôt du droit public.

Dans la mouvance du droit public, se situent les cours d'eau domaniaux dont le lit et l'usage appartiennent à l'Etat qui exerce un pouvoir de police et de gestion, l'usage commun de l'eau étant libre et seule l'utilisation privative du domaine public fluvial est soumise à autorisation.

Dans la mouvance du droit privé, on peut classer :

\begin{abstract}
- les cours d'eau non domaniaux, les propriétaires riverains détenant la propriété du lit et des berges, mais seulement un droit d'usage sur l'eau, dans la limite de la loi et des règlements et autorisations et à charge de la rendre à la sortie du fonds à son cours ordinaire, « de par sa nature, l'eau courante n'étant jamais susceptible de propriété »; en droit strict, cette catégorie d'eau pourrait donc être rattachée aux « res communes »;
\end{abstract}

- les eaux pluviales (non captées), constituent des « res nullius », les eaux stagnantes sans communication avec un cours d'eau non domanial, les eaux de source ne donnant pas naissance à des eaux courantes pleinement régies, dès lors que captées, par le droit privé et assimilables à des «res propriae »;

- à un degré moindre d'emprise du droit privé, compte tenu de l'intervention d'un certain nombre de règlements limitant leur usage, les eaux souterraines, les eaux minérales et thermales, variété tantôt d'eau de source, tantôt d'eaux souterraines.

Les eaux pluviales, encore que ce concept soit assez flou, relèvent plus du droit privé que du droit public. Avant la parution de la loi sur l'eau de 1992, les réglementations les concernant ne sont pas pléthores et renvoient à de multiples codes : code civil, code rural, ...

Les années 1980 représentent une période charnière où la prise de conscience de l'impact des rejets urbains de temps de pluie sur les milieux récepteurs se manifeste. Ce problème crucial est commun à tous les grands pays industrialisés et a conduit la Communauté économique européenne à édicter la directive CEE-91-27I du 21 mai 1991 relative au traitement des eaux urbaines résiduaires.

La Loi N $N^{\circ}$ 2-3 du 3 janvier 1992 sur l'eau et ses textes d'application ont permis, fin 1994, de transcrire en droit français cette directive. Ces textes représentent des avancées significatives :

- les eaux pluviales relèvent de la réglementation de la police des eaux, qu'il s'agisse des rejets eux-mèmes, des ouvrages d'assainissement et leur exploitation et enfin des activités générant ces rejets,

- les collectivités territoriales doivent définir les zones où des mesures doivent être prises pour assurer la maîtrise du ruissellement, qu'il s'agisse là aussi de risque d'inondation ou de lutte contre la pollution. 
Toutefois, qu'il s'agisse d'eaux pluviales ou d'eaux souterraines - considérées comme « res nullius» - seules sont susceptibles d'appropriation celles effectivement recueillies ou captées.

\section{UNE RÉGLEMENTATION ANTÉ- RIEURE ÉCLATÉE ET SOUVENT LAXISTE}

Ainsi les eaux pluviales, encore que cette entité soit assez floue, relèvent elles plus du droit privé que du droit public. Avant la parution de la loi sur l'eau de 1992, les réglementations les concernant ne sont pas pléthores et renvoient à de multiples codes : code civil, code rural, code de la voirie routière, code de la santé publique, code du domaine public fluvial, ...

Annexe A : Réglementation relative à la propriété privée et servitudes inhérentes.

Annexe $B$ : Réglementation relative à la lutte contre les pollutions.

Remarque: Mais la nature (humaine) n'aimant pas le vide, ce relatif déficit d'ordre réglementaire a été partiellement compensé par des textes : circulaire, instruction, ... fixant les «bonnes » pratiques techniques dans la mise en auvre de l'assainissement pluvial:

- circulaire interministérielle $n^{\circ} \mathrm{CG} 1333$ du 22 février 1949 «circulaire Caquot»: instruction technique relative à l'assainissement des agglomérations,

- circulaire du Ministère de la Santé du 10 juin 1976 : assainissement des agglomérations et protection sanitaire des milieux récepteurs,

- instruction technique du Ministère de l'Intérieur $n^{\circ}$ 77.284/INT du 22 juin 1977 relative aux réseaux d'assainissement des agglomérations. Elaborée par la «commission Lonferne », cette instruction se substitue à la circulaire CG 1333.

\section{LA PRISE DE CONSCIENCE DE L'IM- PACT DES RUTP}

Que sont ces «RUTP», ces rejets urbains de temps de pluie ? C'est la prise de conscience que l'on ne peut plus évacuer, au sens propre comme au sens figuré, les eaux pluviales sans se soucier de leurs impacts sur la qualité des milieux récepteurs, rus, rivières ou fleuves dans la plupart des cas.

Le terme même d'eaux pluviales était trompeur, eaux pluviales, eaux de pluie... donc eaux pures. La réglementation antérieure accompagne cette vision des choses; les eaux pluviales strictes, exceptées certaines zones d'activité, échappaient aux procédures d'autorisation préalable. Avec le développement de l'urbanisation, du niveau de confort souhaité par les habitants, avec la multiplication des infrastructures de transport et des sites industriels et commerciaux, la réalité s'est notablement écartée de cette vision idyllique.

L'eau de pluie, synonyme d'eau pure, d'eau salvatrice se trouve elle-même contaminée lorsqu'elle lessive l'atmosphère des miasmes de nos activités urbaines (fumées d'usines, gaz d'échappement des voitures, ...). Elle se charge alors en acidité, en métaux, poussières...

Après avoir lessivé l'atmosphère, elle entreprend le grand nettoyage de nos surfaces imperméabilisées et entraîne dans son ruissellement, sables, feuilles mortes, déjections de nos animaux domestiques mais également une myriade de composés plus sournois accumulés sur la voirie, les parkings, les aires de stockage des commerces et des industries.

A ce stade, les eaux de ruissellement sont confrontées à un cruel dilemme, opter pour le système séparatif ou pour le tout à l'égoût, pardon le système unitaire.

Dans le système unitaire, mélangées aux eaux usées, elles sont définitivement souillées; mais alors que se passe-t-il aux déversoirs d'orage? Pendant longtemps on considéra que, diluant les eaux usées, elles permettaient justement leur rejet en rivière sans impacts nocifs pour celle-ci, si ce n'est quelques flottants, ici ou là. Les mortalités piscicoles en Seine, liées aux orages tombés sur l'agglomération parisienne en juin 1990, mai 1992, juillet 1994 témoignent... les rejets urbains de temps de pluie sont pollués. Les surcharges des stations d'épuration provoquent des problèmes analogues.

Dans le système séparatif, réservant des collecteurs distincts aux eaux usées et aux eaux pluviales, la situation de ces dernières ne devrait pas se dégrader jusqu'à leur rejet en rivière. C'est mal connaître un principe de base de la physique, de la thermodynamique pour être plus précis : l'équilibre d'un système est lié à sa forte entropie. En langage trivial, un réseau séparatif pluvial se stabilise dans le temps quand le désordre de ses branchements devient maximal. Et nous voilà devant un réseau pseudo-unitaire, la capacité hydraulique en moins et les débordements en plus.

Renarque: Heureusement certains gestionnaires de réseaux ont pris conscience de ce problème, mais la tâche est difficile, d'autant plus difficile que les eaux pluviales relèvent du droit privé et les réseaux d'une gestion publique.

Cette prise de conscience n'est pas l'apanage de telle ou telle collectivité qui a pu jouer un rôle précurseur. $\mathrm{Ce}$ problème crucial est commun à tous les grands pays industrialisés souhaitant préserver leurs ressources en eau et leur cadre de vie. A cette fin, la communauté européenne a édicté la Directive CEE-91-271 du 21 mai 1991 relative au traitement des eaux urbaines résiduaires. La France a obligation de répondre à ces exigences en assurant la transcription en droit français, ce qui est effectif aujourd'hui au travers des textes suivants:

- Loi n 92-3 sur l'eau du 3 janvier 1992 modifiée.

- Décrets n 93-742 et 93-743 du 29 mars 1993 modifiés.

- Décret n 94-469 du 3 juin 1994.

- Arrêté du 29 novembre 1994.

- Arrêtés (2) du 22 décembre 1994

\section{LES APPORTS DE LA LOI $N^{\circ}$ 92-3 SUR L'EAU DU 3 JANVIER 1992}

\section{L'unité de la ressource en eau et sa valeur patrimoniale}

L'article $1^{\text {er }}$ de la loi dispose que «l'eau fait partie du patrimoine commun de la nation ». Cette notion de patrimoine commun est à rapprocher de la notion de patrimoine 
du code civil et de la notion de patrimoine commun contenue à l'article L.110 du code de l'urbanisme.

A la notion du patrimoine du code civil est attachée l'idée d'une gestion raisonnable, économe et équilibrée, adaptée aux circonstances de façon à léguer aux générations futures un patrimoine préservé, voire augmenté.

Adapté à l'eau, la notion de patrimoine commun est issue de l'article L.110 du code de l'urbanisme aux termes duquel « le territoire français est le patrimoine commun de la nation. Chaque collectivité publique en est le garant dans le cadre de ses compétences... ». La reconnaissance accordée à l'eau en tant que patrimoine commun ne signifie pas que l'Etat s'approprie l'eau qu'il pourrait gérer, comme s'il s'agissait de son patrimoine ou de son domaine privé, mais vise à renforcer sa légitimité à intervenir en tant que bras séculier de la nation dans l'intérêt général et susceptible de s'opposer à des intérêts particuliers. L'Etat apparaît dès lors essentiellement comme chargé d'un droit de garde.

La nouvelle loi sur l'eau entend également transcender les distinctions physiques de l'eau (superficielle, souterraine, en relation directe ou non avec un cours d'eau) dont certaines avaient pour origine une différenciation juridique (eaux domaniales, eaux non domaniales). Désormais, l'eau est prise en compte pour l'ensemble de son cycle et compte tenu de ses multiples interdépendances et aucune action susceptible d'avoir une incidence sur elle ne se trouve exclue a priori. En effet, aux termes de l'article 10-1, entrent dans le champ d'application de la loi : «les installations, ouvrages, travaux et activités réalisés à des fins non domestiques par toute personne physique ou morale, publique ou privée et entraînant des prélèvements sur les eaux superficielles ou souterraines restitués ou non, une modification du niveau et du mode d'écoulement des eaux ou des déversements, écoulements, rejets ou dépôts directs chroniques ou épisodiques, même non polluants ». La généralité de la prise en compte est manifeste.

La consécration de la nécessité d'une gestion globale et équilibrée

La notion de gestion globale de l'eau n'est pas une idée totalement neuve en France. La loi sur l'eau du 16 décembre 1964 entendait déjà satisfaire ou concilier de multiples exigences et usages reconnaissant la nécessité d'une gestion par bassin hydrographique. La nouvelle loi renforce ces dispositions :

- l'unité de la ressource en eau implique une gestion globale de toutes les catégories d'eau, de façon solidaire, y compris les zones humides,

- l'unité de cette ressource soustend que la gestion globale se préoccupe des aspects qualitatifs mais également quantitatifs,

- la gestion de l'eau ne doit pas s'envisager seulement sous l'angle restrictif des ressources en eau mais également doit aborder la préservation des écosystèmes aquatiques, des sites et des zones humides.

En d'autres termes, aucune gestion ne peut être mise en oeuvre sans que soient prises en compte la ressource en eau, dans son ensemble, et les conséquences de telle activité ou usage sur cette ressource et sur le milieu plus généralement. La gestion est globale car elle doit prendre en compte non seulement la protection de l'eau dans l'instant, mais également sa préservation à long terme et sa restauration.
La loi sur l'eau, ses décrets d'application et les eaux pluviales

Les eaux pluviales, pas plus que les rejets urbains de temps de pluies, n'apparaissent explicitement dans la loi sur l'eau de 1992. Mais leur prise en compte est sous-jacente au travers de la notion de gestion globale «aucune action susceptible d'avoir une incidence sur l'eau ne se trouve $a$ priori exclue ». Les décrets d'application, arrêtés et circulaires sont plus ciblés et traitent explicitement des rejets urbains de temps de pluie.

Annexe $C$ : Police des eaux et rejets urbains de temps de pluie.

Annexe D : L'assainissement des collectivités territoriales.

\section{Références}

[1] Bourlet A. et Garcin J.-L. - Code pratique de l'eau, Editions du Moniteur, $4^{c}$ édition 1991 mise à jour et augmentée.

[2] Sironneau J. - La nouvelle loi sur l'eau ou la recherche d'une gestion équilibrée, RJE de février 1992.

\section{ANNEXE A}

RÉGLEMENTATION RELATIVE À LA PROPRIÉTÉ PRIVÉE ET SERVITUDES INHÉRENTES

\section{Code civil : des servitudes qui découlent de la si-} tuation des lieux

\section{art. 640}

«Les fonds inférieurs sont assujettis envers ceux qui sont plus élevés, à recevoir les eaux qui en découlent naturellement sans que la main de l'homme y ait contribué.

Le propriétaire inférieur ne peut point élever de digue qui empêche cet écoulement.

Le propriétaire supérieur ne peut rien faire qui aggrave la servitude du fonds inférieur. »

\section{art. 641 (loi du 8 avril 1898 )}

« Tout propriétaire a le droit d'user et de disposer des eaux pluviales qui tombent sur son fonds. Si l'usage de ces eaux ou la direction qui leur est donnée aggrave la servitude naturelle d'écoulement établie par l'article 640, une indemnité est due au propriétaire du fonds inférieur... Les maisons, cours, jardins, parcs et enclos attenant aux habitations ne peuvent être assujettis à aucune aggravation de la servitude d'écoulement dans les cas prévus aux paragraphes précédents... ». 
Remarque: La jurisprudence (cours d'Angers 16 mai 1977) précise la notion d'appropriation et des responsabilités qui en découlent.

«L'article 641 du code civil confère seulement à tout propriétaire le droit d'user et de disposer des eaux pluviales qui tombe sur son fonds et, à défaut d'un acte d'appropriation de sa part, ces eaux demeurent sans maître aussi longtemps qu'elles ne font pas l'objet d'une appropriation sur un autre fonds où elles se sont écoulées ou d'une incorporation à un cours d'eau.

Lorsqu’il n'est ni établi ni même allégué qu'un propriétaire a fait un acte d'occupation sur les eaux de ruissellement s'écoulant sur son fonds, qu'il ne leur a pas imprimé une direction différente de la pente naturelle du terrain, qu'il n'en est devenu ni le propriétaire ni le gardien, il ne saurait être tenu responsable... ».

Ainsi, il semblerait que la réalisation d'un réseau public d'assainissement des eaux pluviales soit assimilable à un acte d'appropriation de ces eaux par une collectivité territoriale qui deviendrait alors de fait responsable des nuisances causées par ces eaux.

\section{art. 681}

«Tout propriétaire doit établir des toits de manière que les eaux pluviales s'écoulent sur son terrain ou sur la voie publique; il ne peut les faire verser sur le fonds de son voisin ».

Remarque : La jurisprudence (Cour de cassation chambre civile 11 mai 1976) vient préciser cette obligation.

«Le propriétaire qui laisse, conformément à l'article 681 du code civil, se déverser sur son propre bien ou sur la voie publique, dans les conditions fixées par les règlements de police, les eaux pluviales provenant du toit de son immeuble, n'est pas tenu, après qu'elles aient atteint le sol, de les empêcher de s'écouler selon la pente naturelle du terrain. »

\section{Code de la voirie routière}

Ce code précise certains points marginaux, voire anecdotiques, relatifs aux propriétés bordant une voie routière (voiries nationales, départementales, communales et privées).

\section{art. R. 122-3}

«Les propriétés riveraines des autoroutes ne jouissent du droit de déverser les eaux d'égoût des toitures sur les autoroutes... que sous forme de permissions de voirie... qui peuvent être accordées dans les cas exceptionnels... »

Arrêté du 30 mars 1967, modifié par l'arrêté du 26 août 1974

Refonte de l'instruction générale dans le service des chemins départementaux. Cet arrêté précise les modalités de rejet sur la voirie départementale des eaux pluviales de son fonds ainsi que les obligations faites aux propriétaires riverains d'assurer le libre écoulement des eaux pluviales générées par la voirie.

Loi du 22 juillet 1922 : Assainissement des voies privées

Art. 1 «Les lois et règlements relatifs à l'hygiène des voies publiques et des maisons riveraines de ces voies sont applicables aux voies privées, ...»

\section{Code de l'urbanisme}

Alors que l'urbanisation est régie par les règles de l'urbanisme et que cette urbanisation induit l'imperméabilisation des sols donc les conditions du ruissellement des eaux pluviales, le code de l'urbanisme est peu prolixe sur ce sujet

Contenu du POS (Plan d'Occupation des Sols)

art. L. 123-1 (loi $\mathrm{n}^{\circ} 87.565$ du 22 juillet 1987, modifiée par la loi $n^{\circ}$ 92-3 du 3 janvier 1992 sur l'eau)

«Les plans d'occupation des sols fixent, dans le cadre des orientations des schémas directeurs ou des schémas de secteur, $s$ 'il en existe, les règles générales et les servitudes d'utilisation des sols, qui peuvent notamment comporter l'interdiction de construire... »

\section{art. R. 123-18 (décret $\mathrm{n}^{\circ}$ 87-885 du 30 octobre 1987)}

«...II. Les documents graphiques font apparaître s'il y a lieu :

$3^{\mathbf{c}}$ Les emplacements réservés aux voies et ouvrages publics, aux installations d'intérêt général et aux espaces verts... »

\section{art. R. 123-21 (décret $\mathrm{n}^{\circ} 86-53$ du 10 janvier 1986)}

«Le règlement fixe les règles applicables aux terrains compris dans les diverses zones du territoire couvert par le plan...

$2^{\mathrm{c}}$ Le règlement peut, en outre:

a) édicter les prescriptions relatives à l'accès, à la desserte, à l'équipement en réseaux divers et, le cas échéant, aux dimensions et à la surface des terrains... »

art. R. 123.24 (décret $\mathrm{n}^{\circ} 87-340$ du 21 mai 1987) «Les annexes comprennent:

$1^{\text {er }}$ La liste des emplacements réservés, mentionnés à l'art. R.123-18 (II.3), leur destination, leur superficie et l'indication des collectivités, services et organismes publics bénéficiaires...

$3^{\mathbf{e}}$ les éléments ci-après relatifs aux réseaux d'eau et d'assainissement et au système d'élimination des déchets : schémas et notes techniques... » 
Remarque: Ces articles précisant le contenu des POS traitent notamment de l'assainissement, terme qu'il faut prendre dans son sens général d'assainissement des eaux usées et des eaux pluviales en réseau unitaire ou séparatif, bien que l'article R.123-24 n'étend pas l'objet de la note technique aux ouvrages d'assainissement pluvial.

Permis de construire

\section{art. R. 111-13 (décret $n^{\circ} 77.755$ du juillet 1977)}

«Le permis de construire peut être refusé ou n'être accordé que sous réserve de l'observation de prescriptions spéciales si les constructions, par leur situation ou leur importance imposent, soit la réalisation par la commune d'équipement publics nouveaux hors de proportion avec ses ressources actuelles, soit un surcroît important des dépenses de fonctionnement des services publics. »

\section{ANNEXE B}

\section{RÉGLEMENTATION RELATIVE ì LA LUTTE CONTRE LES POLLUTIONS}

Le règlement sanitaire départemental type (circulaire du 9 août 1978, modifiée par les circulaires des 26 avril 1982. 20 janvier 1983, 18 mai et 10 août 1984) apporte quelques précisions éparses sur l'évacuation des eaux pluviales des locaux d'habitation.

\section{art. 29.1 : évacuation des eaux pluviales}

« Les ouvrages d'évacuation (gouttières, chêneaux, tuyaux de descente) doivent être maintenus en bon état de fonctionnement et d'étanchéité. Ils sont nettoyés autant qu'il est nécessaire et notamment après la chute des feuilles. Il est interdit de jeter des détritus et autres immondices de toute nature dans ces ouvrages... »

\section{art. 42}

«L'évacuation des eaux pluviales et des eaux usées doit pouvoir être assurée en permanence..

Il est interdit d'évacuer des eaux vannes dans les ouvrages d'évacuation d'eaux pluviales et réciproquement... »

\section{art. 100 : salubrité des voies privées}

«... 100.2. Le sol des voies privées, qu'il soit muni ou non d'un revêtement, doit être établi de manière à assurer l'écoulement des eaux, l'entretien facile et une circulation non dangereuse...

Il faut attendre la loi sur l'eau du 16 décembre 1964 pour que soient réglementés ou régis (article 6) les déversements, écoulements, jets, dépôts directs ou indirects d'eau ou de matières, et plus généralement tout fait susceptible d'altérer la qualité de l'eau superficielle ou sou- terraine et des eaux de mer dans les limites temitoriales. La réglementation sur l'eau comporte enfin un volet sur la lutte contre les pollutions.

Des décrets sont venus préciser les modalités d'application de cet article 6 , notamment le décret $n^{\circ} 73-218 \mathrm{du}$ 23 février 1973. La police des eaux ainsi instaurée repose sur le principe de l'autorisation préalable et cette police s'exerce suivant des règles et sur des bases juridiques uniformes aussi bien pour les eaux domaniales que les eaux non domaniales mais également les eaux souterraines.

«L'autorisation détermine les conditions auxquelles les déversements, ... sont subordonnés ». Les autorisations sont subordonnées au respect des prescriptions techniques fixées au niveau national par les arrêtés techniques du 13 mai 1975 et du 20 novembre 1979. La qualité minimale d'un rejet d'effluents urbains a été notamment précisée par la circulaire du 4 novembre 1980.

Le concept d'effluents urbains intègre-t-il les rejets d'eaux pluviales? Oui, mais... l'arrêté du 13 mai 1975 est venu limiter la portée du décret $n^{\circ} 73-218$ en fixant les conditions dans lesquelles certains déversements, écoulements, jets et dépôts de nocivité négligeable sont exemptés de l'autorisation préalable.

Ainsi les rejets d'eaux pluviales strictes sont exemptés dans les 2 cas suivants:

- tous les rejets d'eaux pluviales strictes canalisées autres que ceux provenant d'un établissement industriel, agricole ou commercial ;

- les rejets de tels établissements lorsque leur superficie n'excède pas 1 hectare.

Toutefois, « l'exemption de la formalité d'autorisation ne dispense pas de l'obligation d'obtenir les autorisations prévues par d'autres réglementations ni d'épurer les effluents. Notamment les rejets dans les eaux superficielles et la mer doivent être dépourvus de matières sumageantes, de toute nature, ne pas dégager d'odeurs nauséabondes, ne pas provoquer de coloration visible du milieu récepteur, ne pas être cause de dégradation des abords du point de rejet ou d'ouvrages de toute nature situés dans le milieu récepteur, ne pas porter atteinte à la santé publique ni compromettre l'équilibre biologique du milieu $\gg$.

Aucun maître d'ouvrage n'est censé ignorer la loi, mais cet arrêté interministériel ne permettait pas aux services de police des eaux d'exercer une action préventive que permettait l'autorisation préalable.

Remarque: Si nombre de rejets pluviaux stricts (réseaux séparatifs) échappent à l'autorisation préalable, ce n'est pas le cas des rejets des réseaux unitaires (réseau unique commun aux eaux pluviales et aux eaux usées) que ce soit au droit des déversoirs d'orage ou via la station d'épuration. Or l'expérience passée montre que peu de ces ouvrages ont fait l'objet d'une autorisation préalable.

Dans ce cadre, ils auraient dû satisfaire à l'arrêté du 20 novembre 1979 qui fixe les conditions techniques générales auxquelles sont subordonnées les autorisations délivrées en application du décret $n^{\circ}$ 73-218. L'article 2 précise que l'arrêté d'autorisation fixe pour chaque rejet:

- le débit maximal instantané ;

- le débit moyen qui ne peut être dépassé pendant aucune période de deux heures consécutives;

- le débit moyen qui ne peut être dépassé pendant aucune période de vingt-quatre heures consécutives; 
- le flux moyen de matières polluantes qui ne peut être dépassé pendant aucune période de deux heures consécutives:

- le flux moyen de matières polluantes qui ne peut être dépassé pendant aucune période de vingt-quatre heures consécutives ;

- la qualité minimale de l'effluent.

Les valeurs de ces caractéristiques peuvent être modulées selon les conditions saisonnières.

Mais, là encore, l'article 7 limite la portée de cet arrêté pour les rejets d'eaux pluviales en précisant que l'arrêté d'autorisation peut ne fixer de valeurs limites, que pour certaines des caractéristiques prévues aux articles précédents, dont l'article 2

\section{ANNEXE C \\ POLICE DES EAUX ET REJETS URBAINS DE TEMPS DE PLUIE}

\section{Loi sur l'eau}

art. 8 « Les règles générales de préservation de la qualité et de répartition des eaux superficielles, souterraines et des eaux de la mer dans la limite des eaux territoriales sont déterminées par décret en Conseil d'Etat.

Elles fixent :...

$3^{\circ}$ Les conditions dans lesquelles peuvent être :

- interdits ou réglementés les déversements, écoulements, jets, dépôts directs ou indirects d'eau ou de matière et plus généralement tout à fait susceptible d'altérer la qualité des eaux et des milieux aquatiques...

$5^{\circ}$ Les conditions dans lesquelles sont effectués par le service chargé de la police des eaux... des contrôles techniques des installations, travaux ou opérations... »

Remarque : pas de décrets publiés pour l'application des $8-3^{\circ}$ et $8-5^{\circ}$.

art. 10

«I - Sont soumis aux dispositions du présent article les installations, ouvrages, travaux et activités réalisés à des fins non domestiques pour toute personne physique ou morale, publique ou privée, entraînant... des déversements, écoulements, rejets ou dépôts directs ou indirects, chroniques ou épisodiques, même non polluants. »

«II - Les installations, ouvrages, travaux et activités visés au I sont définis dans une nomenclature établie par décret en Conseil d'Etat... »

Les alinéas III et IV introduisent les notions d'autorisation et de simple déclaration et décrivent les procédures à suivre.

«VII - Les installations et ouvrages existants doivent être mis en conformité avec les dispositions prises en application du II ci-dessus dans un délai de 3 ans à compter de la date de publication de la présente loi. »

Deux décrets ont été pris en application de cet article 10 :
Le décret $n^{\circ}$ 93-742 du 29 mars 1993 relatif aux procédures d'autorisation et de déclaration prévues par l'article 10 de la loi $n^{\circ} 92-3$ du 3 janvier 1992 sur l'eau modifié par les décrets n $94-469$ du 3 juin $1994, n^{\circ} 94-894$ du 13 octobre $1994, n^{\circ} 94-1033$ du 30 novembre 1994 et $n^{\circ} 95-88$ du 27 janvier 1995.

Le décret $n^{\circ}$ 93-743 du 29 mars 1993 relatif à la nomenclature prévue par l'article 10 de la loi $n^{\circ} 92-3$ sur l'eau modifié par le décret n 94-1227 da 26 décembre 1994.

Remarque: Cette nomenclature présente la particularité d'être à plusieurs entrées. Ainsi un même objet peut-il être référencé dans plusieurs rubriques. Dans ce cas, c'est la procédure la plus contraignante qui sera appliquée. Sont donc visés :

- les rejets en eux-mêmes — rubriques 1.2.0, 2.2.0. 3.1.0, 5.1.0, 5.2.0, 5.3.0,

- les activités générant ces rejets - rubriques 2.3.1, $4.1 .0,6.2 .0,6.2 .1,6.4 .0,6.5$

- les ouvrages liés à l'assainissement pluvial et leur exploitation - rubriques 2.6.2, 2.7.0

Remarque: On notera donc qu'une opération d'urbanisme peut relever de la procédure d'autorisation ou de déclaration à plusieurs titres:

- création d'une zone imperméabilisée supérieure à 5 ha d'un seul tenant $\rightarrow$ autorisation,

- rejet d'eaux pluviales dans les eaux superficielles ou dans un bassin d'infiltration, la superficie totale desservie étant supérieure à 20 ha $\rightarrow$ autorisation ou comprise entre 1 et 20 ha $\rightarrow$ déclaration.

- assèchement et imperméabilisation de zone humide $\rightarrow$ autorisation,

- création d'étang ou de plan d'eau, la superficie étant supérieure à 3 ha $\rightarrow$ autorisation ou comprise entre 0,2 et 3 ha $\rightarrow$ déclaration,

- rejet dans les eaux superficielles susceptibles de modifier le régime des eaux, la capacité totale de rejet étant supérieure à $25 \%$ du débit $\rightarrow$ autorisation ou comprise entre 5 et $25 \%$ du débit $\rightarrow$ déclaration. Le débit est le débit de référence (débit quinquennal sec).

\section{ANNEXE D}

\section{L'ASSAINISSEMENT DES COLLECTIVITÉS TERRITORIALES}

\section{Loi sur l'eau}

Titre II. Chapitre $1^{\mathrm{er}}$ - De l'intervention des collectivités territoriales dans la gestion des eaux.

art. $31 \ll \ldots$ les collectivités territoriales et leurs groupements ainsi que les syndicats mixtes.. et la comunauté locale de l'eau sont habilités... pour entreprendre l'étude, l'exécution et l'exploitation de tous travaux, ouvrages ou installations présentant un caractère d'intérêt général ou d'urgence... et visant (notamment) :

- la maîtrise des eaux pluviales et de ruissellement;

- la lutte contre la pollution.» 
Titre II. Chapitre II - De l'assainissement et de la distribution d'eau.

L'article 35 introduit le nouvel article L.372-3 du code des communes qui stipule: « les communes ou leurs groupements délimitent, après enquête publique :

- les zones d'assainissement collectif...,

- les zones relevant de l'assainissement non collectif..., - les zones où des mesures doivent être prises pour limiter l'imperméabilisation des sols et pour assurer la maîtrise du débit et de l'écoulement des eaux pluviales et de ruissellement;

- les zones où il est nécessaire de prévoir des installations pour assurer la collecte, le stockage éventuel et, en tant que de besoin, le traitement des eaux pluviales et de ruissellement lorsque la pollution qu'elles apportent au milieu aquatique risque de nuire gravement à l'efficacité des dispositifs d'assainissement... ».

Remarque: Ces zones peuvent par ailleurs être délimitées dans les POS (art. 38-II modifiant l'art. L. 123-1 du code de l'urbanisme).

Remarque: Ces 2 articles sont importants, le premier introduit la notion de caractère d'intérêt général ou d'urgence qui s'applique à la maîtrise des eaux pluviales et de ruissellement, le second fixe les obligations des communes et de leur groupement dans ce domaine qu'il s'agisse de limiter l'imperméabilisation, de maîtriser les ruissellements ou de lutter contre leur pollution.

L'article 31 a fait l'objet d'un décret d'application :

Décret $\mathbf{n}^{\circ}$ 93-1182 du 21 octobre 1993 relatif à la procédure applicable aux opérations entreprises dans le cadre de l'article 31 de la loi n ${ }^{\circ} 92-3$ du 3 janvier 1992 sur l'eau.

L'article 35 a donné lieu à des textes d'application :
Décret $\mathbf{n}^{\circ}$ 94-469 du 3 juin 1994 relatif à la collecte et au traitement des eaux usées mentionnées aux articles L. 372-1-1 et L. 372-3 du code des communes.

Circulaire du 13 septembre 1994 du Ministre de l'Environnement à Mesdames et Messieurs les Préfets relative à l'assainissement des eaux usées urbaines.

Arrêté du 22 décembre 1994 fixant les prescriptions techniques relatives aux ouvrages de collecte et de traitement des eaux usées mentionnées aux articles L. 372-1-1 et L. 372-3 du code des communes.

Arrêté du 22 décembre 1994 relatif à la surveillance des ouvrages de collecte et de traitement des eaux usées mantionnées aux articles L. 372-1-1 et L. 372-3 du code des communes.

Ces divers textes, décrets et arrêtés, assurent la fin de la transcription de la Directive CEE -91-271 du Conseil des communautés européennes du 21 mai 1991 relative au traitement des eaux urbaines résiduaires en droit français.

Toutefois, le décret susvisé nº 94-469 du 3 juin 1994 ne s'applique explicitement qu'aux eaux usées et à leurs systèmes de collecte et d'épuration. Le problème des eaux pluviales n'est abordé que dans la mesure où elles peuvent perturber le bon fonctionnement de l'assainissement des eaux usées (sous-entendu en système unitaire).

art. 9 «..., les eaux (usées) entrant dans un système de collecte doivent, excepté dans le cas des situations inhabituelles dues à de fortes pluies, être soumises à un traitement... »

art. $19 \ll \ldots$ sont fixées par arrêté... les prescriptions techniques minimales relatives à la police de l'eau qui permettent de garantir, sans entraîner de coût excessif, l'efficacité de la collecte, du transport des eaux et des mesures prises pour limiter les pointes de pollution dues aux précipitations. » 